\title{
LAS TECNOLOGÍAS DE INFORMACIÓN Y COMUNICACIÓN Y SU INFLUENCIA EN EL APRENDIZAJE DE LA ASIGNATURA DE MINERÍA Y MEDIO AMBIENTE EN LOS ESTUDIANTES DE LA ESCUELA ACADÉMICO PROFESIONAL DE INGENIERÍA DE MINAS EN LA UNIVERSIDAD NACIONAL DE HUANCAVELICA - 2017
}

\author{
INFORMATION AND COMMUNICATION TECHNOLOGIES AND \\ THEIR INFLUENCE ON THE LEARNING OF THE COURSE OF \\ MINING AND THE ENVIRONMENT IN THE STUDENTS OF THE \\ PROFESSIONAL ACADEMIC SCHOOL OF MINE ENGINEERING AT \\ THE NATIONAL UNIVERSITY OF HUANCAVELICA - 2017 \\ (D) Felisícimo German Ramírez Rosales ${ }^{*}$, (D) Celia Condori Mendoza ${ }^{1}$, (D) Teodoro Esteban \\ Huamani $^{1}$ \\ ramirezfeli7@hotmail.com; Celiacondori710@gmail.com; Teo.esteban@gmail.com \\ ${ }^{1}$ Universidad Nacional de Huancavelica, Huancavelica, Perú \\ *Correspondencia: Felisícimo German Ramírez Rosales. Email: ramirezfeli7@hotmail.com
}

Recibido: 22.02.2020 | Aprobado: 09.04.2020

\section{RESUMEN}

La formación académica del futuro ingeniero de Minas reviste particular importancia y trascendencia, de ahí nos preguntamos: ¿Qué influencia ejerce la aplicación de las Tecnologías de Información y Comunicación en el aprendizaje de la asignatura de Minería y Medio Ambiente en los estudiantes de la Escuela Académico Profesional de Ingeniería de Minas de la Universidad Nacional de Huancavelica el año 2009?, investigación de carácter cuantitativo, tipo aplicada, nivel explicativo, método experimental, diseño pre experimental. Concluimos que las Tecnologías de Información y Comunicación han mejorado estadísticamente significativo, pedagógica y didácticamente el aprendizaje de la asignatura de Minería y Medio Ambiente.

Palabras clave: Tecnologías de Información y Comunicación, aprendizaje y minería y medio ambiente.

\section{ABSTRACT}

The academic training of future mining engineer and significance of particular importance, hence our research problem says: What influence does the application of Information and Communication Technology in learning the subject of Mining and Environment students Professional Academic School of Mining Engineering of the Universidad Nacional de Huancavelica - 2009, The research is quantitative, applied type, level of explanation, pre-experimental method and experimental design. The nonprobabilistic sample was taken; we conclude that the Information and Communication Technology has significantly improved not only statistically but also pedagogical and educational learning of the subject of Mining and Environment.

Keywords: Information Technologies and Communication, learning and mining and the environment. 


\section{INTRODUCCIÓN}

Sin lugar a dudas las tecnologías de información y comunicación cumple un papel muy importante en la vida del ser humano a nivel mundial, tal es así que necesitamos potenciar el talento humano usando las herramientas de la tecnología de información y comunicaciones de acuerdo a la era en donde vivimos (UNESCO, 2015).

Por otra parte, (Ramirez \& Condori, 2017) menciona que "la paleta de colores del aprendizaje humano es infinitamente variada y matizada, y está además ampliándose. Frente a la intervención del docente, las condiciones del aprendizaje es el componente en el que se realiza el trabajo educativo, pues este aspecto tiene que ver directamente con el uso de las herramientas del internet, es decir las tecnologías de información y comunicación, y en términos más generales la planificación de las sesiones de aprendizaje en función a las TICs en la asignatura de Minería y Medio Ambiente para mejorar o elevar su aprendizaje" (p. 13).

Pero aún con todos estos factores nos quedamos con algunas incertidumbres como por ejemplo como debemos mejorar la calidad del proceso de aprendizaje usando algunas herramientas tecnológicas acorde al avance científico y tecnológica.

Por lo citado ha nacido nuestro problema de la investigación que dice: ¿Qué influencia ejerce la aplicación de las Tecnologías de Información y Comunicación en el aprendizaje de la asignatura de Minería y Medio Ambiente y básicamente nos hemos propuesto el objetivo de determinar la influencia que ejerce la aplicación de las Tecnologías de Información y Comunicación en el aprendizaje de la asignatura de Minería y Medio Ambiente, investigación que se ha realizado con los estudiantes de la Escuela Académico Profesional de Ingeniería de Minas de la Universidad Nacional de Huancavelica-2009.

Si logramos determinar la influencia que ejerce la aplicación de las TICs en el aprendizaje de la asignatura de Minería y Medio Ambiente podremos contribuir con evidencias científicas sobre la influencia de las TICs en el aprendizaje no solo del curso de Minería y Medio ambiente, más bien de resultar favorable esta relación pueden inferirse al aprendizaje en todos los estudiantes de nivel universitario. Por otra parte, esta investigación va a contribuir al acervo de conocimientos científicos sobre la aplicación de los TICs y el aprendizaje.

La aplicación de las TICs viene a ser nuestra variable independiente y el aprendizaje de los estudiantes constituye la variable de estudio dependiente veamos además como otros investigadores abordaron el tema:

Buratto, (2005) concluye "se darán paso a nuevos estándares de aprendizaje con capacitación en la integración de las nuevas tecnologías en la educación y vale decir que la nueva tecnología interactiva es el futuro prometedor de la educación juntamente con la integración de la TIC” (p. 74).

Cruz (2001) afirma que "las tecnologías de información hacen que se da de forma más sencilla y facilitan las cosas a los usuarios que necesitan información ya que atreves de los interfaces de interacción se interactúen y encuentre los necesario en las páginas sociales" (p. 15). 
Porta (2006). Requiere que los "alumnos/as: Dominen el manejo técnico de cada tecnología (conocimiento práctico del hardware y del software que emplea cada medio)" y "Desarrollen un cúmulo de valores y actitudes hacia la tecnología de modo que no se caiga ni en un posicionamiento tecnofóbico (es decir, que se las rechace sistemáticamente por considerarlas maléficas) ni en una actitud de aceptación acrítica y sumisa de las mismas (p. 23).

Además, Sarmiento (2007). Concluye que "El estudio sigue el enfoque interpretativo, desarrolla un modelo de investigación donde integramos metodologías cuantitativas y cualitativas y se basa en un estudio de casos referido a la implementación de un programa de innovación donde los protagonistas son un grupo de docentes y sus alumnos(as)" (p. 782).

Torres (2003), Sus conclusiones fueron: El uso de la plataforma del internet y sus recursos influye en el desenvolvimiento individual y colectivo del estudiante universitario además La utilización de internet se relaciona directa y positiva con el bienestar psicológico de los estudiantes universitarios (p. 113).

\section{METODOLOGÍA}

En esta investigación el diseño general de la investigación viene a ser el pre experimental ya que se considera como útil en investigaciones cualitativas (Kerlinger, et al. 2002 p. 345).

GE: $0_{1} \mathrm{X}_{2}$

Dónde: (X) Aplicación de las TICs.

G.E. Grupo Experimental.

$0_{1}$ : Pre Test

$0_{2}$ : Post Test

La población de estudio estuvo conformada por 252 estudiantes de la carrear de Ingeniería de Minas en el año 2009. De aquí se tienen la muestra no probabilística conformado por 36 estudiantes del IX Ciclo en el año 2009, el cual representa el 14.28\% de la población el cual como dice (Selltiz, 1980, pág. 188) “cumple con los requisitos mínimos del tamaño de muestra (10\%) en el caso de una muestra no probabilística”.

\section{RESULTADOS}

El instrumento para recoger datos fue validado a través del coeficiente de Confiabilidad de la Prueba Escrita del componente curricular de Minería y Medio Ambiente, haciendo uso del Método de Mitades Partidas es igual a 0,938 Ahora bien teniendo como referencia a (Herrera, 1998, pág. 28) los valores hallados pueden ser comprendidos entre la siguiente Tabla: 


\section{Tabla 1}

Niveles de confiabilidad de los instrumentos usados.

\begin{tabular}{ll}
\hline Valores & Tipo de confiabilidad \\
\hline 0,53 a menos & Confiabilidad nula \\
0,54 a 0,59 & Confiabilidad baja \\
0,60 a 0,65 & Confiable \\
0,66 a 0,71 & Muy Confiable \\
0,72 a 0,99 & Excelente confiabilidad \\
1.0 & Confiabilidad perfecta \\
\hline
\end{tabular}

Como se obtuvo 0,938 en la confiabilidad del pilotaje respectivo, se concluye afirmando que la Prueba Escrita del componente curricular de Minería y Medio Ambiente tiene una excelente confiabilidad.

Por otra parte vimos la validez de los instrumentos tal es así, Manheim y Rich (1991), citado por (Ramirez \& Condori, 2017, pág. 28) menciona: "Validez es el término que nosotros usamos para referirnos al alcance en el cual nuestras medidas corresponden a los conceptos que ellas están tratando de reflejar", y para (Hernandez, Fernadez, \& Baptista, 2010, pág. 277) "La validez, en términos generales, se refiere al grado en que un instrumento mide la variable que pretende medir... La validez es una cuestión más compleja que debe alcanzarse en todo instrumento de medición que se aplica"; por ello hemos sometido a Juicio de Expertos por (5) profesionales de reconocida trayectoria en la investigación y docencia universitaria), todos ellos con grados académicos de Doctor. De las fichas de evaluación del juicio de experto se ha podido apreciar que las puntuaciones cuantitativamente oscilan entre el 73\% y $96 \%$, por lo tanto, se afirma que dicho instrumento es válido para ser aplicado.

Ahora apreciemos el Coeficiente de la Validez de Criterio, medido mediante el alfa de Cronbach, el cual también está estandarizado de 0 a 1, trabajando con las desviaciones estándar parciales y totales de las fichas de validez de contenido se ha calculado el mencionado coeficiente. Como quiera, que para que los ítems sean válidos se necesita un completo acuerdo entre los jueces el alfa de Cronbach calculado es igual a 0,952 con lo que se concluye afirmando que el instrumento Prueba Escrita del componente curricular de Minería y Medio Ambiente posee una excelente validez.

Para obtener los datos se han realizado un pre y post test a la muestra de la población de estudio y se obtuvieron los siguientes datos:

\section{Tabla 2}

Datos obtenidos al emplear los instrumentos de recolección de datos

\begin{tabular}{ccc}
\hline Alumnos & Pre test & Pos test \\
\hline 1 & 6 & 17 \\
2 & 12 & 18 \\
3 & 3 & 14 \\
4 & 5 & 12 \\
5 & 4 & 8 \\
6 & 9 & 19 \\
7 & 8 & 13
\end{tabular}




\begin{tabular}{|c|c|c|}
\hline 8 & 8 & 9 \\
\hline 9 & 10 & 15 \\
\hline 10 & 5 & 15 \\
\hline 11 & 12 & 14 \\
\hline 12 & 3 & 8 \\
\hline 13 & 8 & 17 \\
\hline 14 & 5 & 8 \\
\hline 15 & 14 & 18 \\
\hline 16 & 3 & 12 \\
\hline 17 & 6 & 14 \\
\hline 18 & 3 & 12 \\
\hline 19 & 5 & 14 \\
\hline 20 & 6 & 12 \\
\hline 21 & 7 & 11 \\
\hline 22 & 12 & 17 \\
\hline 23 & 14 & 18 \\
\hline 24 & 9 & 15 \\
\hline 25 & 5 & 9 \\
\hline 26 & 8 & 15 \\
\hline 27 & 10 & 16 \\
\hline 28 & 9 & 14 \\
\hline 29 & 4 & 10 \\
\hline 30 & 13 & 17 \\
\hline 31 & 3 & 8 \\
\hline 32 & 12 & 18 \\
\hline 33 & 7 & 14 \\
\hline 34 & 9 & 14 \\
\hline 35 & 8 & 14 \\
\hline 36 & 7 & 12 \\
\hline
\end{tabular}

Fuente: Elaboración propia.

\section{Gráfico 1}

Comparativo (Pre Testy Post Test)

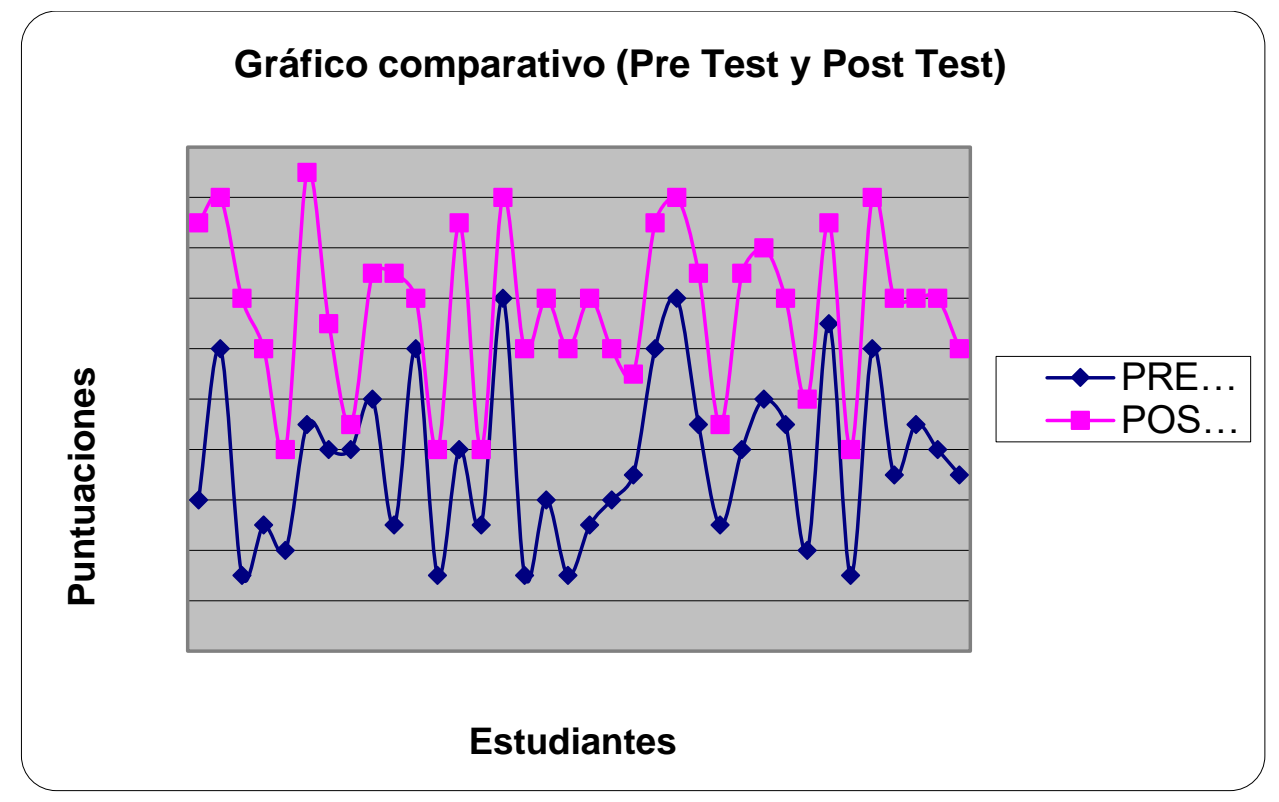

Fuente: Elaboración propia. 


\section{Tabla 3}

Resumen estadístico entre el pre test y el post test

\begin{tabular}{lll}
\hline Resumen Estadístico & & \\
\hline Estadígrafos & Pre Test & Post Test \\
Media Aritmetica & 7,56 & 13,64 \\
Mediana & 7,50 & 14,00 \\
Moda & 3,00 & 14,00 \\
Punt. max. & 14 & 19 \\
Punt. min. & 3 & 8 \\
Rango & 11 & 11 \\
Desv. stad. & 3,31 & 3,24 \\
Coef. de var. & 43,78 & 23,78 \\
Kurtosis & $-0,8146641$ & $-0,8068727$ \\
Asimetría & 0,0503902 & $-0,3339581$ \\
R de Pearson & 0,7249083 & \\
\hline
\end{tabular}

Fuente: Elaboración propia.

Como se puede apreciar en el cuadro resumen, los calificativos que se obtuvieron del pre test y post test son bien diferenciados. Mientras que en el pre test los alumnos tienen una media aritmética de 7,56. El cual es bastante bajo, en el post test se tiene 13,64 (6.08 puntos más) es decir han mejorado significativamente. Respecto a la mediana, en el pre test tenían 7,50, y en el post test tienen 14,00 (6,50 puntos más). Respecto a la moda en el pre test era 3,00, y en el post test tienen 14,00.

El puntaje máximo en el pre test fue 14 mientras que en el post test fue 19 (5,00 puntos más) y respecto al puntaje mínimo en el pre test fue 3 mientras que en el post test fue 8 (5,00 puntos más).

El rango que es el recorrido del menor al mayor puntaje, en el pre test fue 11 mientras que en el post test también fue 11 (o sea fueron iguales).

Respecto a la Desviación Estándar en el pre test fue 3,31 mientras que en el post test fue 3,24, el cual nos dice que los resultados son ligeramente un poco más homogéneos en el pre test que en el post test.

Respecto a la Kurtosis, en ambos casos se tiene puntuaciones negativas (Pre Test $=-0,814)$ y (Post Test $=-0,806)$, o sea tienen gráfica platicúrtica y en cuanto se refiere a la Asimetría, en el pre test es 0,050 el cual significa una Asimetría positiva o a la derecha y en el por test se tiene -0,333 el cual significa una Asimetría negativa o a la izquierda.

Y finalmente respecto a la " $r$ " de Pearson, entre los resultados del pre test y post test se tiene una $\mathrm{r}=0,724$, el cual nos dice que existe una relación directa y positiva entre los resultados de éstas dos evaluaciones. 


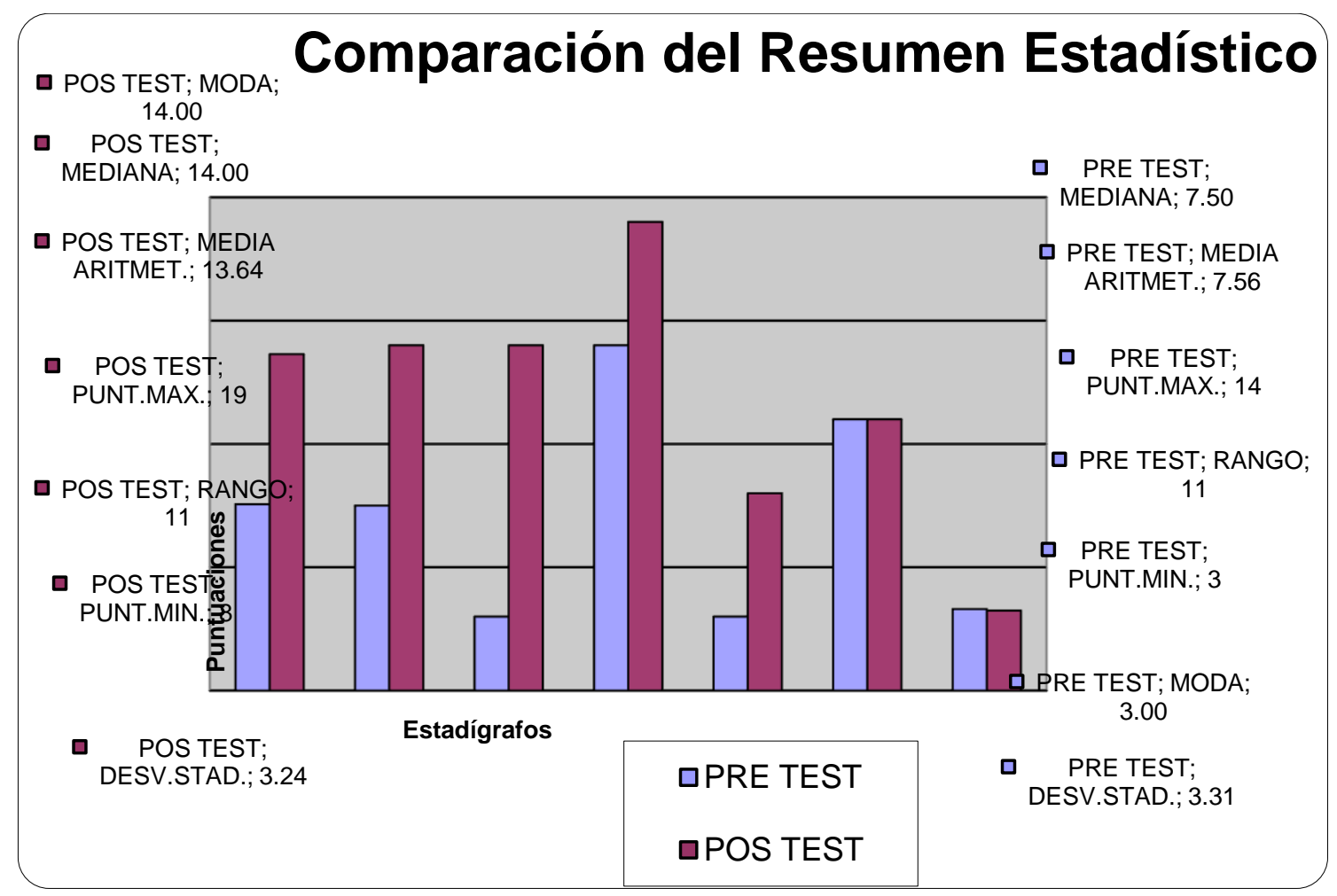

Fuente: Elaboración propia.

\section{CONTRASTACIÓN DE LAS HIPÓTESIS ESPECÍFICAS}

Primera hipótesis específica

La primera hipótesis específica dice: Los factores de carácter pedagógico condicionantes de las Tecnologías de Información y Comunicación en los estudiantes de la Escuela Académico Profesional de Ingeniería de Minas de la Universidad Nacional de Huancavelica-2009 se presentan de manera divergente.

En esta parte se ha podido evidenciar que los factores condicionantes del uso de las Tecnologías de Información y Comunicación en los estudiantes de la Escuela Académico Profesional de Ingeniería de Minas de la Universidad Nacional de Huancavelica-2009 se presentan de manera divergente, ya que son muchos los factores que influyen en su uso, entre gellos el factor acceso a las TICS, aunque este fenómeno se ha ido superando gradualmente ya que la EAP de Ingeniería de Minas para este año 2009 se ha implementado en cuanto a los recursos informáticos, asimismo el factor docente, ya que este a cada instante motiva a los estudiantes en cuanto a su uso adecuado y oportuno; asimismo el factor económico, ya que los recursos económicos influyen en el uso de las TICs, y también se tiene el uso cada vez más intensivo de las herramientas de internet (correo electrónico, chat, navegación, entre otros); con lo cual se prueba la primera hipótesis específica en todo su sentido y significancia.

Segunda hipótesis específica

La segunda hipótesis específica dice: Los niveles de aprendizaje en la asignatura de Minería y Medio Ambiente en los estudiantes de la Escuela Académico Profesional de Ingeniería de Minas de la Universidad Nacional de Huancavelica-2009 es bajo y/o regular. 
En esta parte, base a los cuadros y gráficos $\mathrm{N}^{\circ} 01$ y 02 ; se ha podido apreciar que los niveles de aprendizaje en el pre test del componente curricular de Minería y Medio Ambiente, son realmente bajos y en algunos casos regulares, ya que se tiene una media de 7,56. Respecto a la mediana se tiene 7,50 y una moda de 03 ; bajo rendimiento académico que dificulta comportamiento y aprendizaje de estos alumnos.

Con esta información obtenida, tenemos la suficiente evidencia para afirmar que la segunda hipótesis específica, también se contrasta en todo su sentido y extensión ya que los niveles de aprendizaje de los estudiantes del IX ciclo en el componente curricular de Minería y Medio Ambiente de la Escuela Académico Profesional de Ingeniería de Minas de la Universidad Nacional de Huancavelica - 2009 es bajo y/o regular.

\section{Tercera hipótesis específica}

La tercera hipótesis específica dice: Existe una relación directa y positiva entre de la asignatura de Minería y Medio Profesional de Ingeniería de Minas de la Universidad Nacional de Huancavelica-2009.

Para esta prueba hemos utilizado el Coeficiente de Correlación con un nivel de significancia del $0.05 \%$, específicamente la " $r$ " de Pearson, entre los resultados en ambos grupos (Pre Test y Post Test), obteniéndose una $r=0,724$, el cual nos dice que existe una relación directa y positiva con lo cual también se prueba esta tercera hipótesis específica de investigación.

\section{CONTRASTACIÓN DE LA HIPÓTESIS GENERAL}

Sin dejar de lado otros planteamientos, se ha optado por seguir los siguientes pasos para el contraste de la hipótesis:

- Formular la hipótesis nula y alterna de acuerdo al problema.

- Escoger un nivel de significancia o riesgo $\alpha$.

- Escoger el estadígrafo de prueba más apropiado.

- Establecer la región crítica.

- Calcular los valores de la prueba estadística de una muestra aleatoria de tamaño n.

- Rechazar la Ho si el estadígrafo tiene un valor en la región crítica y no rechazar (aceptar) $=$ en el otro caso.

\section{Planteamiento de Hipótesis}

\section{Hipótesis Nula}

$$
H_{o}: \mu G E=\mu G C
$$

Ho: "No existe diferencias significativas de aprendizaje del grupo de alumnos que trabajó con las Tecnologías de Información y Comunicación con respecto al grupo al cual no se le aplicó dichas Tecnologías de Información y Comunicación”.

Lo que para la investigación significa: "La aplicación de las Tecnologías de Información y Comunicación no influyen significativamente en el aprendizaje de la asignatura de Minería y Medio Ambiente en los estudiantes de la Escuela Académico Profesional de Ingeniería de Minas de la Universidad Nacional de Huancavelica el año 
2009", por lo tanto, no se observan diferencias significativas entre la aplicación de la Prueba Escrita en el Pre Test y el Post Test.

\section{Hipótesis Alterna}

$H_{1}: \mu G E \neq \mu G C$

H1: "Existe diferencias significativas de aprendizaje del grupo de alumnos que trabajó con las Tecnologías de Información y Comunicación con respecto al grupo al cual no se le aplicó dichas Tecnologías de Información y Comunicación”.

\section{Nivel de significancia o riesgo}

Se eligió el nivel de significancia, es decir el riesgo que se asume acerca de rechazar la Ho cuando en realidad debe aceptarse por ser verdadera. El nivel de significancia utilizado en el diseño cuasi experimental fue $\alpha=0,05$; por ser adecuado en las investigaciones en Ciencias Sociales y Pedagógicas Sierra, (2003).

\section{El estadígrafo de prueba:}

El estadígrafo de prueba, apropiado para la presente investigación fue la Prueba Z, por lo que la muestra en estudio fue mayor que 30; además como los datos tienen distribución normal porque $\mathrm{AS}_{(2)}=-0,334$ es pequeñísima, además que las varianzas muestrales de los grupos estudiados (Pre Test y Post Test) son semejantes. La prueba Z sigue una distribución con el número de observaciones del grupo Experimental.

Además, como en la hipótesis alterna $\left(\mathrm{H}_{1}\right)$ existe dos posibilidades $\left(H_{1}: \mu_{1}>\mu_{2} o ́\right.$ $\left.\mu_{1}<\mu_{2}\right)$ se aplicó la prueba bilateral, o sea a dos colas.

\section{Valor crítico y regla de Decisión:}

Para la prueba de dos colas con $\alpha=0,05$ en la tabla, tenemos para el lado derecho el valor crítico $Z=1,96$. Y por simetría al lado izquierdo se tiene también: $Z=-1,96$.

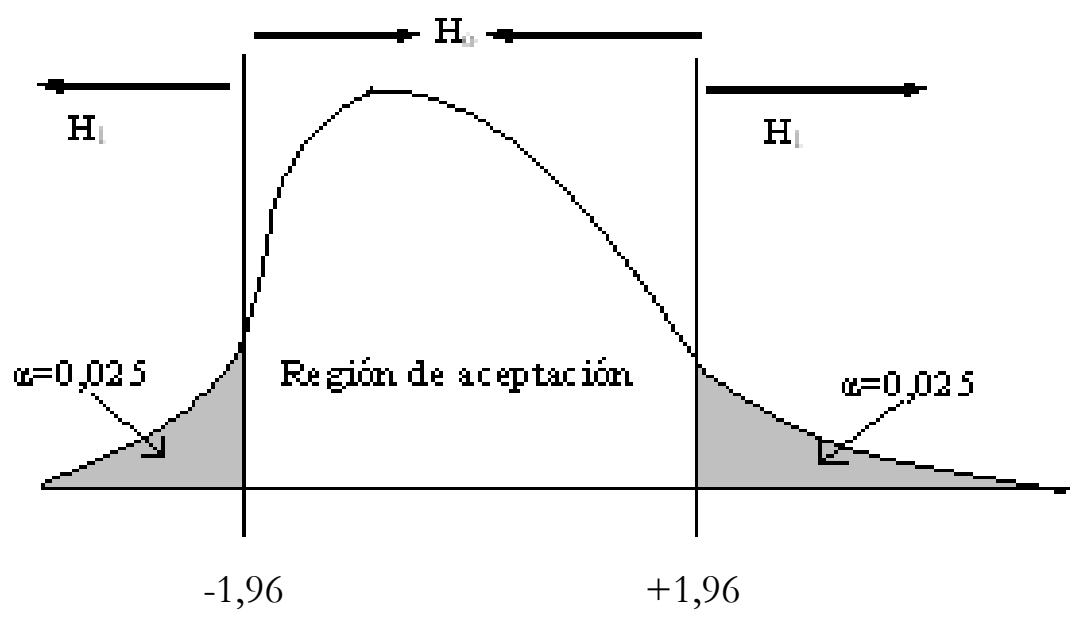

Regla de decisión: rechazar la hipótesis nula si: $Z \geq 1,96$ ó $Z \leq-1,96$. 


\section{Cálculo del Estadígrafo de Prueba:}

$$
Z_{c}=\frac{\bar{X}_{1}-\bar{X}_{2}}{\sqrt{\frac{S_{1}^{2}}{n_{1}}+\frac{S_{2}^{2}}{n_{2}}}}=\frac{7,56-13,64}{\sqrt{\frac{3,31^{2}}{36}+\frac{3,24^{2}}{36}}}=-5,085
$$

\section{Decisión Estadística:}

Como la $Z$ calculada $\left(Z_{c}\right)$ con los datos procesados es igual a: $\mathbf{- 5 , 0 8 5}$ y este es mayor que -1,96; por lo tanto, cae en la zona de rechazo; entonces se rechaza la $\mathrm{H}_{\mathrm{o}}$, y se acepta la hipótesis alterna $\mathrm{H}_{1}$.

\section{DISCUSIÓN Y RESULTADOS}

Para poder interpretar en forma adecuada, precisa y completa nuestros resultados, debemos partir determinando los datos obtenidos antes de ejecutar las Tecnologías de Información y Comunicación en el componente curricular de Minería y Medio Ambiente en los estudiantes de la Escuela Académico Profesional de Ingeniería de Minas de la Universidad Nacional de Huancavelica el año 2009.

Es necesario empezar refiriendo que, conforme se verificó en la sección de presentación de datos, los niveles de aprendizaje del conjunto de la muestra de estudiantes (36) del IX ciclo de la Escuela Académico Profesional de Ingeniería de Minas de la Universidad Nacional de Huancavelica el año 2009, fueron realmente muy bajos en los inicios del semestre académico 2009-I, es decir antes de aplicar las Tecnologías de Información y Comunicación para mejorar su aprendizaje.

En esos iniciales bajos niveles de aprendizaje en los estudiantes, no solamente habrían influido los consabidos, factores o causas extra curriculares (económicas, culturales, psicológicos, sociales, estructurales, históricas, etc.), sino que también han influido de modo notable, múltiples factores de carácter estructuralmente pedagógico didáctico, entre los más importantes: la mayoría de los estudiantes en referencia tuvieron dificultades en acceder a las TICs, pero como se explicó hojas atrás, este problema gradualmente se ha ido solucionando. Asimismo, se tiene el factor docente, ya que este siempre ha ido promoviendo y fomentando el uso de las TICs en el proceso de aprendizaje de manera adecuada y oportuna.

Asimismo, también se ha podido analizar que, de acuerdo a los datos obtenidos, la gran mayoría de los docentes no usan las diversas Tecnologías de Información y Comunicación durante el desarrollo de sus asignaturas (componentes curriculares), debido al desconocimiento y limitado acceso a éstas; y solo una minoría de dichos profesores ejecutan prácticas relacionadas al mismo.

El hecho de que casi todos los estudiantes del IX ciclo de la Escuela Académico Profesional de Ingeniería de Minas de la Universidad Nacional de Huancavelica el año 2009, hayan iniciado el semestre académico con un bajo nivel de aprendizaje (rendimiento académico) y no solo en el componente curricular de Minería y Medio Ambiente, sino en casi todas las demás - conforme lo indican los datos presentados- es corroborado con los 
estadígrafos de tendencia central y dispersión el cual menciona que en el Pre Test los resultados obtenidos cuantitativamente son bajos.

Asimismo, los resultados obtenidos con nuestra investigación son novedosos y útiles pues evidencian que, como producto de nuestro experimento, se ha logrado una diferencia estadísticamente significativa de medias en el Pre Test y el Pos Test $(0,009)$, teniendo en cuenta que sus medias numéricas fueron 7,56 y 13,64 respectivamente; con tendencia a 0,000. Diferencias significativas que nos lleva a rechazar la hipótesis nula (Ho), y por ende, a aceptar la hipótesis alterna (Hi) propuesta en la presente investigación.

Y además como la $\mathrm{Z}$ calculada $(\mathrm{Zc})$ con los datos procesados es igual a $-5,085$ y este cae en la zona de rechazo; entonces se rechaza la Ho, y se acepta la hipótesis alterna H1, que sostiene lo siguiente: "Existe diferencias significativas de aprendizaje del grupo de estudiantes que trabajó respecto al Pre Test".

Tales resultados nos permitieron inferir que las Tecnologías de Información y Comunicación, consistente exclusivamente en el uso de las herramientas del internet, pues el nivel de significancia entre estos fue de 0,009. Asimismo, en comparación a la situación inicial y posterior a la ejecución de las sesiones de aprendizaje que duró el experimento, siendo en este caso el nivel de significancia tendiente a 0,000 , es decir es mayor que en el caso anterior.

\section{CONCLUSIONES}

1. Los niveles de aprendizaje de los estudiantes de la Escuela Académico Profesional de Ingeniería de Minas de la Universidad Nacional de Huancavelica en el año 2009 fueron muy bajos al iniciar el semestre académico 2009-I, ya que la mayoría absoluta de ellos tuvieron puntuaciones entre 03 y 14; bajos niveles que expresaban y explicaban las diversas dificultades que adolecían de Minería y Medio Ambiente.

2. Los bajos niveles de aprendizaje de los estudiantes se explican por factores de carácter pedagógico y didáctico, entre ellos el más importante, el limitado y en algunos casos.

3. Después de aplicar las Tecnologías de Información y Comunicación se constató aprendizaje del Post Test, es decir cuando se recibió el tratamiento experimental, con respecto al Pre Test, es decir cuando no se aplicó dicho tratamiento; puesto que el nivel de significancia entre estas dos evaluaciones fue de 0,009 , es decir hubo diferencias estadísticamente significativas entre sus medias.

4. Las Tecnologías de Información y Comunicación desarrolla las capacidades el año 2009, ya que viabiliza y familiariza al estudiante con el uso de las principales herramientas del internet, así como también con la solución de problemas de contexto realista, facilitando su interpretación y transferencia a los conocimientos nuevos.

\section{RECOMENDACIONES}

1. Se debe incluir en los Planes Curriculares de Estudio de las diferentes carreras profesionales de Ingeniería, preseminarios, seminarios y/o talleres que utilicen las Tecnologías de Información y Comunicación, a fin de superar o remediar las 
diversas dificultades que se evidencian en el bajo aprendizaje de los estudiantes del sistema universitario.

2. Se debe proponer a las instancias académicas pertinentes de la Escuela Académico Profesional de Ingeniería de Minas de la Universidad Nacional de Huancavelica (Coordinaciones, Departamentos Académicos, la Comisión de Acreditación, y la Decanatura) el diseño de políticas de capacitación y actualización docente basadas en los principios y técnicas.

3. Al planificar la ejecución de las Tecnologías de Información y Comunicación, prever la inclusión de los guiones relacionadas con los contenidos de los textos que integran los contenidos temáticos de las diferentes áreas, haciéndolo así más productivo, adecuado y cabal esta estrategia de enseñanza.

4. Replicar la presente investigación en otras Facultades de Ingeniería de universidades públicas y privadas; de sus resultados y conclusiones.

\section{REFERENCIAS BIBLIOGRÁFICA}

Buratto, C. (2005). La Informática como Recurso Pedagógico - Didáctico en la Educación Secundaria. Argentina.

Cruz, C. (2001). Planificación de sistemas y tecnologías de la información. Cabimas.

Hernandez, R., Fernadez, C., \& Baptista, M. D. (2010). Metodología de la Investigación (Vol. Quinto). Mexico: Mc Graw Hill.

Herrera, C. (1998). Criterios de validez de instrumentos en la investigación cientifica. Lima: Nuevo Perú.

Porta, R. (2006). Una Nueva Educación para un Nuevo Siglo. La Habana.

Ramirez, F., \& Condori, C. (2017). Las Tecnologias De Información Y Comunicación Y Su Influencia En El Aprendizaje De La Asignatura De Minería Y Medio Ambiente En Los Estudiantes De La Escuela Académico Profesional De Ingeniería De Minas En La Universidad Nacional De Huancavelica - 2017. Huancavelica.

Sarmiento, M. (2004). La enseñanza de las matemáticas y las NTIC. Tarragona.

Selltiz, C. (1980). Métodos de la Investigación enlas relaciones sociales. Madrid: Rialp.

Torres, W. (2003). Utilización del internet y e bienestar psicológico en estudiantes Universitarios de Alto y Bajo nivel de acceso a la Tecnología de la Inofrmación por computadora. Lima.

UNESCO. (15 de 02 de 2015). Las TIC en la educación. Obtenido de Unesco: https://es.unesco.org/themes/tic-educacion

\section{CITAR COMO:}

Ramírez Rosale, F. G., Condori Mendoza, C., \& Esteban Huamani, T. (2020). Las tecnologías de información y comunicación y su influencia en el aprendizaje de la asignatura de minería y medio ambiente en los estudiantes de la escuela académico profesional de ingeniería de minas en la Universidad Nacional de Huancavelica - 2017. Puriq, 2(2), 127-138. https://doi.org/10.37073/puriq.2.2.77 